\title{
On existence, uniqueness, and numerical evaluation of solu- tions of ordinary and hyperbolic differential equations $\left(^{*}\right),\left({ }^{* *}\right)$ :
}

By J. B. Diaz (a Maryland, U.S.A.).

Summary. - After a preliminary survey of related results, a general uniqueness theorem for the ordinary differential equation $\frac{d y}{d x}=f(x, y)$ is given in section 4. The general uniqueness theorem for the hyperbolic partial differential equation $u_{x y}=f(x, y, u)$, proved in section 5, is an exact analogue of the general uniqueness theorem for the ordinary differential equation $\frac{d y}{d x}=f(x, y)$.

\section{Introduction.}

This paper is concerned with the initial value problem: $\frac{d y}{d x}=f(x, y)$, $y\left(x_{0}\right)=y_{0}$, and the characteristic boundary value problem :

$u_{n y}=f\left(x, y, u, u_{x}, u_{y}\right), \quad u\left(x_{0}, y\right)=\tau(y), \quad u\left(x, y_{0}\right)=\sigma(x), \quad \sigma\left(x_{0}\right)=\tau\left(y_{0}\right)$

The initial value problem for the ordinary differential equation $\frac{d y}{d x}=f(x, y)$ has been treated by many different methods; of which PIOARD's method of suecessive approximations and the EULER-CAUCHY polygon method are perhaps the best known. The EULER-CAUCHY polygon method is of special importance in the numerical evaluation of solutions. Section 2 is devoted to an exposition of the application of the method of successive approximations and the polygon method in order to obtain existence and uniqueness theorems for the initial valne problem for the ordinary differential equation $\frac{d y}{d x}=f(x, y)$.

(*) This research was supported in part by the United States Air Force through the Air Force Office of Scientific Research and Development Command under Contract No. AF $49(638)-228$.

(**) This is a detailed account of lectures given at the Sixth Conference of Arsenal Mathematicians, held at Duke University Durham, North Carolina, June 1, 2, 1960, and at the Symposium on the Numerical Treatment of Ordinary Differential Equations, Integra! and Integro-Differential Equations, Rome, 20-24 Seprember 1960. 
The method of successive approximations is readily available for the characteristic boundary value problem for the hyperbolic partial differential equation $u_{x y}=f\left(x, y, u, u_{x}, u_{y}\right)$ (see PIOARD [1]). For the purpose of nume. rical computation, as well as for theoretical reasons, the devising of an exact analogue of the EULER-CAUCHY polygon method appeared to be desirable (see, e. g. G. ZwIRner [5], J. B. DIaz [11]). Section 3 contains an exposition of the application of the method of successive approximations and the analogue of the EULER-CAUCHY polygon method in order to obtain existence and uniqueness theorems for the characteristic boundary value problem for the hyperbolic partial differential equation $u_{x y}=f\left(x, y, u, u_{x}, u_{y}\right)$. For further developments, in the ease of the characteristic boundary value problem, reference is made to R. H. Moone [16]. and P. Santoro [17]; and in the case of the CAUCHY and the mixed boundary value problems to JaMES CONLAN [14].

A more general aniqueness theorem for the initial value problem for the ordinary differential equation $\frac{d y}{d x}=f(x, y)$ is proved in section 4. The argument is patterned after that given by J. B. DiAz and W. L. WALTER [15]. The method of proof followed is believed to be simpler than those usually employed in proving uniqueness theorems; it also furnishes more information than the usual results.

Finally, section 5 contains the proof of a more general uniqueness theorem for the characteristic boundary value problem for the hyperbolio partial differential equation $u_{x y}=f(x, y, u)$. This equation is dealt with, rather than the full equation $u_{x y}=f\left(x, y, u, u_{x}, u_{y}\right)$, solely in order to carry out, step by step, the complete analogy with the reasoning of the theorem of section 4 for the ordinary differential equation $\frac{d y}{d x}=f(x, y)$.

A more complete bibliography is to be found in J. B. Diaz [11]. The present bibliography consists, for the most part, of references which have appeared since the publication of [11].

\section{Existence and uniqueness theorems for the ordinary differential equa-} tion $\frac{d y}{d x}=f(x, y)$; Picard's method of successive approximations and the Euler-Cauchy polygon method. - The classical initial value problem for the ordinary differential equation

$$
\frac{d y}{d x}=f(x, y)
$$

(where the real valued continuous function $f(x, y)$ is defined for $x_{0} \leqq x \leqq x_{0}+a$ and $-\infty<y<+\infty)$ consists in the determination of a real valued function 
J. B. DIAz: On existence, uniqueness, and numerical evaluation, etc.

$y(x)$. defined on $x_{0} \leqq x \leqq x_{0}+a$, which satisfies the given ordinary differential on this interval, and also satisfies the initial condition

$$
y\left(x_{0}\right)=y_{0}
$$

where $y_{0}$ is a given real number.

Among the many methods which have been employed for proving the existence of a solution $y(x)$ to this problem, mention will be made here only of PICARD's method of successive approximations and of the EULER-CAUCHY polygon method.

Two olassical theorems concerning this problem are the following:

ThEorem I. - If

(1) the real valued function $f(x, y)$ is defined for all $(x, y)$ such that

$$
x_{0} \leqq x \leqq x_{0}+a, \quad-\infty<y<+\infty,
$$

where $x_{0}$ and $a$ are real numbers, with $a>0$, and $f(x, y)$ is centinuous and bounded in absolute value, so that for a certain non-negative constant $M$ one has

$$
|f(x, y)| \leqq M
$$

for all these $(x, y)$; and $f$ satisfies a Lmpschitz condition in the argument $y$ (that is, there is a constant $L \geqq 0$ such that

$$
\left|f(x, y)-f\left(x, y_{1}\right)\right| \leqq L\left|y-y_{1}\right|
$$

whenever $x_{0} \leqq x \leqq x_{0}+a$, and $\left.-\infty<y, y_{1}<+\infty\right)$;

(2) $y_{0}$ is a real number;

then

(3) there is one and only one real valued function $y(x)$ defined on the interval $x_{0} \leqq x \leqq x_{0}+a$, which has a finite derivative throughout this interval, and satisfies the ordinary differential equation

$$
\frac{d y}{d x}(x)=f(x, y(x))
$$

on $x_{0} \leqq x \leqq x_{0}+a$, and the initial condition $y\left(x_{0}\right)=y_{0}$.

Theorem 2. - If

(1) the real valued function $f(x, y)$ is defined for all $(x, y)$ such that

$$
x_{0} \leqq x \leqq x_{0}+a, \quad-\infty<y<+\infty
$$


where $x_{0}$ and $a$ are real numbers, with $a>0$, and $f(x, y)$ is continuous and bounded in absolute value, so that for a certain non-negative constant $M$ one has

$$
|f(x, y)| \leqq M
$$

for all these $(x, y)$;

(2) $y_{0}$ is a real number ;

then

(3) there is at least one real valued function $y(x)$ defined on the interval $x_{0} \leq x \leqq x_{0}+a$, which has a finite derivative thronghout this interval, and satisfies the ordinary differential equation

$$
\frac{d y}{d x}(x)=f(x, y(x))
$$

on $x_{0} \leqq x \leqq x_{0}+a$, and the initial condition $y\left(x_{0}\right)=y_{0}$.

In theorem 1, both the existence and the uniqueness of a solution $y(x)$ are asserted, for continuous functions $f(x, y)$ which satisfy a LiPscHITz condition in $y$, as specified. Theorem 1 is usually proved by means of Proard's method of successive approximations, which may be briefly described as follows. A sequence of functions $y_{n}(x), n=0,1,2, \ldots$, continuous on the interval $x_{0} \leqq x \leqq x_{0}+a$, is defined by means of successive integrations:

$$
\begin{aligned}
& y_{0}(x) \equiv y_{0}, \\
& y_{n}(x)=y_{0}+\int_{x_{0}}^{x} f\left(t, y_{n-1}(t)\right) d t, \quad n=1,2, \ldots .
\end{aligned}
$$

It is then shown, using the LIPschitz condition satisfied by $f(x, y)$, that the following inequality holds on the interval $x_{0} \leqq x \leqq x_{0}+a$ for $n=1,2, \ldots$

$$
\left|y_{n+1}(x)-y_{n}(x)\right| \leqq L \int_{x_{0}}^{x}\left|y_{n}(t)-y_{n-1}(t)\right| d t
$$

which implies the uniform convergence of the sequence of continuous functions $y_{n}(x)$ to a continuous limit function $y(x)$ on this interval. This function $\dot{y}(x)$ may now be readily shown to be a solution of the problem. That there is no other solution ( dition again. For if $y(x)$ is a solution and $z(x)$ is also a solution, then one 
has that

$$
|y(x)-z(x)| \leqq L \int_{x_{0}}^{x}|y(t)-z(t)| d t, \quad x_{0} \leqq x \leqq x_{0}+a,
$$

which yields readily that $y \equiv z$.

In theorem 2, only the existence of a solution $y(x)$ is asserted, but not its uniqueness, while only the continuity of the function $f(x, y)$, but not a Lipschitz condition, is assumed. The argument given above, using the LIPSCHITz condition, is not applicable to show existence or to show uniqueness. Indeed, uniqueness does not hold in general. Example: consider the ordinary differential equation $\frac{d y}{d x}=|y|^{\frac{1}{2}}$, with the initial condition $y(0)=0$, on the interval $0 \leqq x \leqq 1$; the functions $y(x) \equiv 0$ and $z(x)=(1 / 4) x^{2}$ are distinct solutions. (Of course, in order to comply with the requirement of boundedness of the absolnte value, the function $t$ must be defined suitably, e. g., $f(x, y)=|y|^{1}$ for $|y| \leqq 1 / 4$ and $f(x, y)=1 / 2$ for $\left.|y|>1 / 4\right)$. However, existence of a solution may be shown by means of the so-called EuLERCAUCHY polygon method.

For the numerical purpose of the actual construction of a solution, the EULER-CAUCHY polygon method is usually the most advantageons. The con. struction of the EULER-CAUCHY polygons may be described as follows. For each positive integer $m$, let

$$
x_{0} \equiv x_{0, m}<x_{1, m}<x_{2, m}<\ldots<x_{m-1, m}<x_{m, m} \equiv x_{0}+a,
$$

be a subdivision of the interval $x_{0} \leqq x \leqq x_{0}+a$ into $m$ closed subintervals $x_{k, m} \leqq x \leq x_{k+1, m}$, where $k=0,1, \ldots, m-1$. On each subinterval the ordinary differential equation is, so to speak, replaced by one whose right-hand side is a (suitable chosen) constant, so that the corresponding function approximating a solution turns out to be a linear function on each subinterval. More precisely put, the polygonal function $y_{m}$, which is an approximation to a solution, is defined recurrently by the equations

$$
\begin{array}{ccc}
\frac{d y_{m}}{d x}(x)=f\left(x_{0, m} ; y_{0}\right), & y_{m}\left(x_{0, m}\right)=y_{0}, & \text { on } x_{0, m} \leqq x \leqq x_{1, m}, \\
\frac{d y_{m}}{d x}(x)=f\left(x_{1, m} ; y_{1}\right) . & y_{m}\left(x_{1, m}\right)=y_{1}, & \text { on } x_{1, m} \leqq x \leqq x_{2, m}, \\
\ldots & \ldots & \ldots \\
\frac{d y_{m}}{d x}(x)=f\left(x_{k, m} ; y_{k}\right), & y_{m}\left(x_{k, m}\right)=y_{k}, & \text { on } x_{h, m} \leqq x \leqq x_{k+1, m},
\end{array}
$$


for $k=0,1, \ldots, m-1$. Notice that, for simplicity in writing these equations, the symbol $y_{k}$ is used to denote the value of the function $y_{m}(x)$ at $x_{k, m}$, a value which is obtained from the definition of $y_{m}$ as a linear function on the preceding subinterval $x_{k-1, m} \leqq x \leqq x_{k, m}$ and which is used as an initial value for the function $y_{m}(x)$ for the "miniature» initial value problem (of the same kind as the original one, but whose differential equation has a constant right hand side):

$$
\frac{d y_{m}}{d x}(x)=f\left(x_{k, m} ; y_{k}\right), \quad y_{m}\left(x_{k, m}\right)=y_{k},
$$

on the next subinterval $x_{k, m} \leqq x \leqq x_{k+1}, m$. For each positive integer $m$, the function $y_{m}(x)$ is continuous on the interval $x_{0} \leq x \leq x_{0}+a$, but its derivative will, in general, not exist throughout the interval, since it may jump at the subdivision numbers $x_{k, m}$.

Under the sole additional hypothesis that the function $f(x, y)$ is bounded in absolute value on $x_{0} \leqq x \leq x_{0}+a,-\infty<y<+\infty$, it follows that the sequence of functions $\left\{y_{m}(x)\right\}$ is equibounded in absolnte value and equi continuous on the interval $x_{0} \leqq x \leq x_{0}+a$; and hence, by AscolI's theorem, there is a subsequence $\left\{y_{m}(x)\right\}$ which converges uniformly to a continuous limit function on $x_{0} \leqq x \leqq x_{0}+a$. If, further, it is supposed that the maximum length of the subintervals of the subdivision of $x_{0} \leqq x \leqq x_{0}+a$ approaches zero, i. e.

$$
\lim _{m \rightarrow \infty}\left[\max _{k=0,1, \ldots, m-1}\left(x_{k+1, m}-x_{k, m}\right)\right]=0
$$

then every such gontinnous limit function is a solution of the original initial value problem, whose solution need not be unique. (The condition on the maximum length of the subintervals is automatically satisfied when the $m^{\text {th }}$ subdivision consists of $m$ subintervals of equal length, namely $a / m$ ). If, besides this, the function $f(x, y)$ satisfies a LIPScHInz condition with respect to $y$, i. e. there is a number $L \geq 0$ such that

$$
\left|f(x, y)-f\left(x, y_{1}\right)\right| \leqq L\left|y-y_{1}\right|,
$$

whenever $x_{0} \leqq x \leqq x_{0}+a$, and $-\infty<y, y_{1}<+\infty$, then the whole sequence $\left\{y_{m}(x)\right\}$ converges uniformly on $x_{0} \leqq x \leqq x_{0}+a$ to the (known to be unique) solution of the original initial value problem.

REMARK. - Actually, theorem 1 remains valid when it is not required that $f(x, y)$ be bounded in absolute value. But this boundedness assumption is essential for the validity of theorem 2, if one insists upon seeking solutions which are defined "in the large», i. e. on the entire interval $x_{0} \leqq x \leqq x_{0}+a$ : 
J. B. Didz: On existence, uniqueness, and numerical evaluation, etc.

EXAMPLE. - Consider the ordinary differential equation $\frac{d y}{d x}=y$, with the initial condition $y(0)=1$, on the interval $0 \leqq x \leqq 2$, which has a solution $y(x)=1 /(1-x)$. Notice that, by means of an argument involving theorem 1 , the solution is uniquely determined on any interval $0 \leqq x \leqq a<1$.

3. Existence and uniqueness theorems for the hyperbolic partial diflerential equation $u_{x y}=f\left(x, y, u, u_{x}, u_{y}\right)$; Picard's method of successive approximations and the analogue of the Euler-Cauchy polygon method. The classical «characteristic» boundary value problem for the hyperbolic partial differential equation

$$
u_{x y}=f\left(x, y, u, u_{x}, u_{\nu}\right)
$$

(where the real-valued continuous function $f(x, y, z, p, q)$ is defined for all $(x, y, z, p, q)$ satisfying

$$
\left.x_{0} \leq x \leqq x_{0}+a \quad y_{0} \leq y \leq b, \text { and }-\infty<z, p, q<+\infty\right)
$$

consists in the determination of a real-valued function $u(x, y)$ which satisfies the given parial differential equation on the rectangle $x_{0} \leqq x \leqq x_{0}+a$ ) $y_{0} \leqq y \leqq y_{0}+b$, and also satisfies the conditions

$$
\begin{array}{ll}
u\left(x, y_{0}\right)=\sigma(x), & \text { for } x_{0} \leqq x \leqq x_{0}+a, \\
u\left(x_{0}, y\right)=\tau(y), & \text { for } y_{0} \leq y \leq y_{0}+b,
\end{array}
$$

where $\sigma\left(x_{0}\right)=\tau\left(y_{0}\right)$ and $\sigma(x)$ and $\tau(y)$ are given continuously differentiable functions on the characteristics $y=y_{0}$ and $x=x_{0}$ of the given hyperbolic equation.

Two known theorems coucerning this boundary value problem, which "correspond», in the theory of the hyperbolic partial differential equation $u_{x \nu}=f\left(x, y, u, u_{x}, u_{\nu}\right\rangle$, to the two theorems discussed in section 2 for the ordinary differential equation $\frac{d y}{d x}=f(x, y)$, are the following :

Theorem 3. - If

(1) the real-valued function $f(x, y, z, p, q)$ is defined for all $(x, y, z, p . q$ such that

$$
x_{0} \leqq x \leqq x_{0}+a, \quad y_{0} \leqq y \leqq y_{0}+b, \quad-\infty<z, p, q<+\infty,
$$

(where $x_{0}, y_{0}, a, b$ are real numbers, with $a>0, b>0$ ) and is continuous and bounded in absolute value, so that for a certain non-negative constant $M$ one has

$$
|f(x, y, z, p, q)| \leqq M
$$


for all these $(x, y, z, p, q)$; and $f$ satisfies a LIPschitz condition in the three arguments $z, p, q$ (that is, there is a constant $L \geqq 0$ such that one has

$\left|f(x, y, z, p, q)-f\left(x, y, z_{1}, p_{1} q_{1}\right)\right| \leqq L\left|z-z_{1}\right|+L\left|p-p_{1}\right|+L\left|q-q_{1}\right|$,

for any $(z, p, q)$ and $\left(z_{1}, p_{1}, q_{1}\right)$, whenever $(x, y)$ lies in the rectangle

$$
\left.R: \quad x_{0} \leqq x \leqq x_{0}+a, \quad y_{0} \leqq y \leqq y_{0}+b\right) ;
$$

(2) the real-valued function $\sigma(x)$ is defined for all $x$ such that $x_{0} \leqq x \leqq x_{0}+a$ and possesses a continuous first derivative $\sigma^{\prime}(x)$ for all these $x$, while the real-valued function $\tau(y)$ is defined for all $y$ such that $y_{0} \leqq y \leqq y_{0}+b$ and possesses a continuous first derivative for all these $y$; and $\sigma\left(x_{0}\right)=\tau\left(y_{0}\right)$;

then

(3) there is one and only one real-valued function $u(x, y)$ defined on the rectangle $R$, which is continuous together with its partial derivatives

$$
\frac{\partial u}{\partial x}, \frac{\partial u}{\partial y}, \frac{\partial^{2} u}{\partial x \partial y}\left(=\frac{\partial^{2} u}{\partial y \partial x}\right), \text { on } R,
$$

satisfies the partial differential equation

$$
\frac{\partial^{2} u}{\partial x \partial y}(x, y)=f\left(x, y, u(x, y), \frac{\partial u}{\partial x}(x, y), \frac{\partial u}{\partial y}(x, y)\right), \quad \text { for }(x, y) \text { in } R
$$

and the characteristic conditions

$$
\begin{array}{ll}
u\left(x, y_{0}\right)=\sigma(x), & \text { for } x_{0} \leqq x \leqq x_{0}+a \\
u\left(x_{0}, y\right)=\tau(y), & \text { for } y_{0} \leqq y \leqq y_{0}+b .
\end{array}
$$

THEOREM 4. - If

(1) the real-valued function $f(x, y, z, p, q)$ is defined for all $(x, y, z, p, q)$ such that

$$
x_{0} \leqq x \leqq x_{0}+a, \quad y_{0} \leqq y \leqq y_{\mathrm{v}}+b, \quad-\infty<z, p, q<+\infty,
$$

where $x_{0}, y_{0}, a, b$ are real numbers, with $a>0, b>0$, and is continuous and bounded in absolute value, so that for a certain non-negative constant $M$ one has

$$
|f(x, y, z, p, q)| \leqq M
$$


J. B. Diaz: On existence, uniqueness, and numerical evaluation, etc.

for all these $(x, y, z, p, q)$; and $f$ satisfies a LIPSchrtz condition in the two arguments $p ; q$ that is, there is a constant $L \geqq 0$ such that one has

$$
\left|f(x, y, z, p, q)-f\left(x, y, z, p_{1}, q_{1}\right)\right| \leqq L\left|p-p_{1}\right|+L\left|q-q_{1}\right|
$$

for any $(p, q)$ and $\left(p_{1}, q_{2}\right)$, whenever $(x, y)$ lies in the rectangle

$$
R: x \leqq x \leqq x_{0}+a, \quad y_{0} \leqq y \leqq y_{0}+b,
$$

and $z$ is any real number);

(2) the real-valued function $\sigma(x)$ is defined for all $x$ such that $x_{0} \leqq x \leqq x_{0}+a$, and possesses a continuous first derivative $\sigma^{\prime}(x)$ for all these $x$, while the real-valued function $\tau(y)$ is defined for all $y$ such that $y_{0} \leqq y \leqq y_{0}+b$, and possesses a continuous first derivative for all these $y$; and $\sigma\left(x_{0}\right)=\tau\left(y_{0}\right)$;

then

(3) there is at least one real-valued function $u(x, y)$ defined on the rectangle $R$ which is continuous together with its partial derivatives $\frac{\partial u}{\partial x}$, $\frac{\partial u}{\partial y}, \frac{\partial^{2} u}{\partial x \partial y}\left(=\frac{\partial^{2} u}{\partial y \partial x}\right)$ on $R$, satisfies the partial differential equation

$$
\frac{\partial^{2} u}{\partial x \partial y}(x, y)=f\left(x, y, u(x, y), \frac{\partial u}{\partial x}(x, y), \frac{\partial u}{\partial y}(x, y)\right), \text { for }(x, y) \text { in } R
$$

and the characteristic conditions

$$
\begin{array}{ll}
u\left(x, y_{0}\right)=\sigma(x), & \text { for } x_{0} \leqq x \leqq x_{0}+a, \\
u\left(x_{0}, y\right)=\tau(y), & \text { for } y_{0} \leqq y \leqq y_{0}+b .
\end{array}
$$

In theorem 3 , both the existence and the uniqueness of a solution $u(x, y)$ are asserted, for continuous functions $f(x, y, z, p q)$ which satisfy a LIPscHITz condition in $z, p, q$, as specified. The proof of theorem 3 , by successive approximations, goes back to PICARD [1], and may be briefly described as fo'lows. A sequence of functions $u_{n}(x, y), n=0,1,2, \ldots$, continuous on the rectangle $R$, is defined by means of successive integrations:

$$
\begin{aligned}
& u_{0}(x, y) \equiv \sigma(x)+\tau(y)-\sigma\left(x_{0}\right) \\
& u(x, y)=\sigma(x)+\tau(y)-\sigma\left(x_{0}\right) \\
& +\int_{x_{y_{0}}}^{x} f\left(s, t, u_{n-1}(s, t), \frac{\partial u_{n-1}}{\partial x}(s, t), \frac{\partial u_{n-1}}{\partial y}(s, t)\right) d s d t
\end{aligned}
$$


for $n=1,2, \ldots$. It is then shown, using the LIPSoHitz condition, that this sequence of continuous functions converges uniformly on the rectangle $R$ to a continuous limit function $u(x, y)$, which turns out to be a solution of the boundary value problem in question. That there is no other solution is then proved by using the LIPSCHITz condition again.

In theorem 4, only the existence of a solution is asserted, but not its uniqueness, while only the continuity of the function $f(x, y, z, p, q)$ and a LrPSCHITz condition in $p, q$, but not a LIPSCHITz condition in $z, p, q$, is assumed. The argument given above, using the Lipschitz condition in $z, p, q$. is not applicable to show existence or to show uniqueness. Indeed, uniqueness does not hold in general (see P. HaRTMan and A. Wintren $[6$, p. 84] and P. LeEHEY [4, p. 23]). Example: consider the differential equation $u_{x y}=|u|^{\frac{1}{2}}$, and the characteristic conditions $u(x, 0)=0$, for $0 \leqq x \leqq a$, and $u(0, y)=0$, for $0 \leqq y \leqq b$, which has as solutions both $u_{1}(x, y) \equiv 0$ and $u_{2}(x . y)=\frac{1}{16} x^{2} \dot{y}^{2}$, on the rectangle $0 \leqq x \leqq a, 0 \leqq y \leqq b$. (Of course $f$ must be suitably defined; compare the similar situation at the end of section 2). P. HaRTMan and A. Wintwer [6] have also given an example of a continuous $f(x, y, z, p, q)$ for which the boundary value problem in question does not possess a solution. Theorem 4 was first proved by $P$. LeEHex [4] and P. Hartmann and A. Wintiner [6], by different methods.

A proof of theorem 4 by means of an analogue of the EULER-CAUOHY polygon method was given in J. B. DIAZ [11]. This process may be briefly described as follows. For each pair of positive integers $m$ and $n$, consider the following subdivisions of the intervals $x_{0} \leqq x \leqq x_{0}+a$ and $y_{0} \leqq y \leqq y_{0}+b$ :

$$
\begin{aligned}
& x_{0} \equiv x_{0, m}<x_{1, m}<x_{2, m}<\ldots<x_{m-1, m}<x_{m, m} \equiv x_{0}+a, \\
& y_{0} \equiv y_{0, n}<y_{1, n}<y_{2, n}<\ldots<y_{n-1, n}<y_{n, n} \equiv y_{0}+b,
\end{aligned}
$$

which produce a subdivision of the rectangle $R$. The miniature problem in the present method (which is a boundary value problem of the same kind as the original one, but with a differential equation having a constant right hand side; compare with the similar situation for the ordinary differential equation $\frac{d y}{d x}=f(x, y)$ in section 2$)$ is just :

$$
\begin{aligned}
& \frac{\partial^{2} u_{m n}}{\partial x \partial y}(x, y)=A_{k \lambda}, \quad \text { for } x_{k} \leqq x \leqq x_{k+1}, \quad y_{\lambda} \leqq y \leqq y_{\lambda+1} \\
& u_{m n}\left(x, y_{\lambda}\right)=D_{k \lambda}+B_{k \lambda}\left(x-x_{k}\right), \quad \text { for } x_{k} \leqq x \leqq x_{k+1} \\
& u_{m n}\left(x_{k}, y\right)=D_{k \lambda}+C_{k \lambda}\left(y-y_{\lambda}\right), \quad \text { for } y_{\lambda} \leqq y \leqq y_{\lambda+1}
\end{aligned}
$$


where $A_{k \lambda}, B_{k \lambda}, C_{k \lambda}$, and $D_{k \lambda}$ are suitable constants, depending on the subrectangle (for simplicity in writing, $x_{k}$ his been written for $x_{k, m}$ and $y_{\lambda}$ for $y_{n, n}$ in the formulation of the boundary value problem for the subrectangle). This means that on each subrectangle the approximating function $u_{m}$ is bilinear in $(x, y)$, i. e. it is a hyperbolic paraboloid:

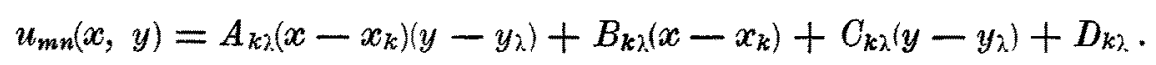

This process just described reduces essentially, in the special case of the equation $u_{x y}=f(x, y, u)$ and equal subdivisions of the $x$ and $y$ intervals, to the process given by $G$. ZWIRnER [5, pp. 222-223], who did not consider the more general equation $u_{x y}=f\left(x, y, u, u_{x}, u_{y}\right)$. Similar methods, analogous to the one just described, have been employed to prove existence theorems for the same boundary value problem by P. Hartman and A. Wintner [6], R. H. Moore [8], and R. Conti [10], but they do not appear to be as convenient for numerical purposes as the one just described, which requires knowledge only of the tabulated values of the given functions $\sigma(x)$ and $\tau(y)$ (from which the difference quotients may easily be calculated) and does not require the tabulated values of the first derivatives $\sigma^{\prime}(x)$ and $\tau^{\prime}(y)$. Mention is also made of different, but closely related method, also analogous to the EuLER-CaUCHY polygon method, given ly H. LEWy [2] (see also H. BeOKERT (3]) for the solution of the initial value problem for second order quasilinear partial differential equations in two independent variables, which appears to require more differentiability assumptions than the present method.

REMARK. - Actually, theorem 3 remains valid when it is not required that $f(x, y, z, p, q)$ is bounded in absolute value, call this the «extended theorem $3 »$. But this boundedness assumption is essential for the validity of theorem 4, if one insists upon seeking solutions which are defined in the large, i. e. on the entire rectangle $R$. Example: consider the equation $u_{x \nu}=u^{2}$, with the characteristic conditions $u(x, 0)=1, u(0, y)=1$, on the square $0 \leqq x \leqq 2,0 \leqq y \leqq 2$, which has as solution $u(x, y)=\frac{1}{(1-x)(1-y)}$. Notice that, by an argument involving theorem 3 , the solution is uniquely letermined on every rectangle $0 \leqq x \leqq a<1,0 \leqq y \leqq b<1$.

\section{A more general uniqueness theorem for the ordinary diflerential} equation $\frac{d y}{d x}=f(x, y)$. - This uniqueness theorem for the initial value problem $\left.\frac{d y}{d x}=f(x, y), y^{\prime} x_{0}\right)=y_{0}$ was given in J. B. Draz and W. L. Walter [15]. The method of proof (which involves only simple properties of continuous real valued functions and the ordinary LAGRANGE mean value theorem 
of the differential calculus) appears to be simpler and more direct than that used in textbooks, which-in general-rely upon the theory of the definite integral to some extent. Furthermore, not only are the hypotheses concerning $f(x, y)$ fewer here, but also the differential equation $\frac{d y}{d x}=f(x, y)$ is not re. quired to hold at all at $x=x_{0}$ or at $x=x_{0}+a$, so that the theorem given here is more general than that usually given compare with the classical theorems 1 and 2 of section 2 \%.

THEOREM 5. - If

(1) the real-valued function $f(x, y)$ is defined for all $(x, y)$ such that

$$
x_{0}<x<x_{0}+a, \quad-\infty<y<+\infty \text {, }
$$

where $x_{0}$ and $a$ are real numbers, with $a>0$. and $f$ satisfies a LIPschitz condition in the argument $y$ (that is, there is a constant $L \geqq 0$ such that

$$
\left|f(x, y)-f\left(x, y_{1}\right)\right| \leqq L\left|y-y_{1}\right|,
$$

whenever $x_{0}<x<x_{0}+a$, and $\left.-\infty<y, y_{1}<+\infty\right)$;

(2) $y_{0}$ is a real number;

then

(3) there is at most one real valued function $y(x)$, defined and conti. nuous on the interval (half-open) $x_{0} \leq x<x_{0}+a$. which has a finite derivative on $x_{0}<x<x_{0}+a$, and satisfies the ordinary differential equation

$$
\frac{d y}{d x}(x)=f(x, y(x))
$$

on $x_{0}<x<x_{0}+a$. and the initial condition $y\left(x_{0}\right)=y_{0}$.

Proof. - Suppose that $u(x)$. is a "solution» and that $v x$ is also a «solution». Then one has $u^{\prime}(x)=f(x, u(x))$ and $v^{\prime}(x)=f(x, v(x)$ on $x_{0}<x<x_{0}+a$, with $u(x)$ and $v(x)$ continuous on $x_{0} \leqq x<x_{0}+a$, and $u\left(x_{0}\right)=v\left(x_{0}\right)=y_{0}$. Suppose, contrary to what one wants to prove, that the function $u(x)-v(x)$ (which is known to be continuous on $x_{0} \leqq x<x_{0}+a$, to be finitely differentiable on $x_{0}<x<x_{0}+a$ and to vanish at $x=x_{0}$ ) does not vanish throughout $x_{0} \leqq x<x_{0}+a$. Then there exists a number $\xi$ with $x_{0} \leqq \xi<x_{0}+a$, such that the function $u(x)-v(x)$ is not identically zero on any $x$ interval $\xi \leqq x \leqq \xi+\lambda<x_{0}+a$, where $\lambda>0$. (Indeed, $\xi$ is just the maximum of all numbers $\xi_{0}$ such that $u(x)-v(x)=0$ whenever $x_{0} \leqq x \leqq \xi_{0}$ ). Choose $\lambda>0$ so small that $\lambda L<1$, where $L$ is the LIPschitz constant of 
$f(x, y)$. Since the absolute value function $\mid u(x)-v(x)$ is continuous and not identically zero on the closed interval $\xi \leqq x \leqq \xi+\lambda$, it bas a positive maximum at some number, call it $\xi_{m}$, of this interval (notice that $\xi<\xi_{m}$ ). Now. applying first the mean value theorem of the differential calculus to the function $u-v$ on the interval $\xi \leqq x \leqq \xi_{m}$, and then using the LiPSCHITz condition for $f$, one obtains, in succession, that

$$
\begin{aligned}
0<\left|u\left(\xi_{m}\right)-v\left(\xi_{m}\right)\right| & =\left|\left[u\left(\xi_{m}\right)-v\left(\xi_{m}\right)\right]-[u(\xi)-v(\xi)]\right| \\
& =\left|\xi_{m}-\xi\right|\left|u^{\prime}\left(\xi^{*}\right)-v^{\prime}\left(\xi^{*}\right)\right| \\
& =\left(\xi_{m}-\xi\right)\left|f\left(\xi^{*}, u\left(\xi^{*}\right)\right)-f\left(\xi^{*}, v\left(\xi^{*}\right)\right)\right| \\
& \leqq \lambda L\left|u\left(\xi^{*}\right)-v\left(\xi^{*}\right)\right| \\
& \leqq \lambda L\left|u\left(\xi_{m}\right)-v\left(\xi_{m}\right)\right|
\end{aligned}
$$

where $\xi^{*}$ is the "mean value abscissa", satisfying $\xi<\xi^{*}<\xi_{m} \leqq \xi+\lambda$; and thus

$$
1 \leqq \lambda L
$$

contradicting the initial choice of $\lambda$.

The theorem just proved is applicable in cases where the usual theorems (see theorms 1 and 2 of section 2) are powerless. Example: eonsider the initial value problem consisting of the ordinary differential equation $\frac{d y}{d x}=\mathrm{c}(x) y$, where $c(x)$ is an arbitrary real valued function defined on $x_{0}<x<x_{0}+a$, which is, hovever, bounded in absolute value (i. e. there exists a number $L \geqq 0$ such that $|c(x)| \leqq L$ for $\left.x_{0}<x<x_{0}+a\right)$; and the initial condition $y\left(x_{0}\right)=0$. The function $y(x) \equiv 0$ is clearly a «solution»; it is continuous on $x_{0} \leqq x<x_{0}+a$ and satisfies the differential equation on $x_{0}<x<x_{0}+a$. Here, $f(x, y)=c(x, y$, but theorems 1 and 2 of section 2 are not applicable, since $c(x)$ need not be continuous. On the other hand, theorem 5 still guarantees that there is no other «solution».

5. A more general uniqueness theorem for the hyperbolic partial differential equation $u_{x y}=f(x, y, u)$. - The present section contains a more general uniqueness theorem for the partial differential equation $u_{x y}=f(x, y, u)$. Although a similar result is valid for $u_{x y}=f\left(x, y, u, u_{x}, u_{y}\right)$, the simpler equation $u_{, y}=f(x, y, u)$ is dealt with because in the argument for the other equation there is a slight complication which obscures somewhat the analogy with the corresponding theorem of section 4 for $\frac{d y}{d x}=f(x, y)$. 
Theorem 6. - If

(1) the real-valued function $f(x, y, z)$ is defined for all $(x, y, z)$ such that

$$
x_{0}<x<x_{0}+a, \quad y_{0}<y<y_{0}+b, \quad-\infty<z<+\infty,
$$

where $x_{0}, y_{0}, a, b$ are real numbers. with $a>0, b>0$, and $f$ satisfies a LrpsonITz condition in the argument $z$ (that is, there is a constant $L \geqslant 0$ such that

$$
\left|f(x, y, z)-f\left(x, y, z_{1}\right)\right| \leqq L: z-z_{x} \mid,
$$

whenever $x_{0}<x<x_{0}+a, y_{0}<y<y_{0}+b$, and $-\infty<z, z_{1}<+\infty$ );

(2) the real-valued function $\sigma(x)$ is defined for all $x$ such that $x_{0} \leqq x<x_{0}+a$ and the real-valued function $\tau(y)$ is defined for all $y$ such that $y_{0} \leqq y<y+b$; then

(3) there is at most one real-valued function $u\left(x, y_{\text {; }}\right.$, defined and continuous on the (partly open) rectangle $x_{0} \leqq x<x_{0}+a, y_{0} \leqq y<y_{0}+b$, which has a finite, continuous partial derivative $u_{x}$ on the (partly open) rectangle $x_{0}<x<x_{0}+a, y_{0} \leqq y<y_{0}+b$, has a finite mixed partial derivative $u_{x y}$ on the (open) rectangle $x_{0}<x<x_{0}+a, y_{0}<y<y_{0}+b$, and satisfies the partial differential equation

$$
u_{x y}(x, y)=f(x, y, u(x, y))
$$

on the (open) rectangle $x_{0}<x<x_{0}+a, y_{0}<y<y_{0}+b$.

(It should be noticed that the lack of symmetry of the hypotheses with respect to $x$ and $y$ arises because, in the partial differential equation $\left.u_{x y^{\prime}} x, y\right)=f(x, y, u(x, y))$, the mixed partial derivative is taken first with respect to $x$ and then with respect to $y$, in that order. Nothing is assumed here about the existence of the mixed partial derivative in the opposite order, $u_{y x}$; or, for that matter, of the first order partial derivative $u_{\nu}$ ).

Proof. - Suppose that $u(x, y)$ is a ssolution » and that $v(x, y)$ is also a «solution». Then one has $u_{x y}(x, y)=f(x, y, u(x, y))$ and $v_{x y}(x, y)=f(x, y, v(x, y))$ on $x_{0}<x<x_{0}+a, y_{0}<y<y_{0}+b$; the partial derivatives $u_{x}$ and $v_{x}$ are finite and continuous on $x_{0}<x<x_{0}+a, y_{0} \leqq y<y_{0}+b$; and the partial derivatives $u_{x y}, v_{x y}$ are finite on $x_{0}<x<x_{0}+a, y_{0}<y<y_{0}+b$. Further, one has that $u\left(x, y_{0}\right)=v\left(x, y_{0}\right)=\sigma(x)$ for $x_{0} \leqq x<x_{0}+a$ and that $u\left(x_{0}, y\right)$ $=v\left(x_{0}, y\right)=\tau(y)$ for $y_{0} \leqq y<y_{0}+b$. Suppose, contrary to what one wants to prove, that the function $u(x, y)-v(x, y)$ (which is known to be continuous on $x_{0} \leqq x<x_{0}+a, y_{0} \leqq y<y_{c}+b$; to possess a finite, continuous partial derivative with respect to $x$ on $x_{0}<x<x_{0}+a, y_{0} \leqq y<y_{0}+b$, and a finite partial derivative with respect to $x y$ on $x_{0}<x<x_{0}+a, y_{0}<y<y_{0}+b$; and 
to vanish when $x=x_{0}$ and when $y=y_{0}$ ) does not vanish throughout $x_{0} \leqq x<x_{0}+a, \quad y_{0} \leqq y<y_{0}+b$. Then there exists a point $(\bar{x}, \bar{y})$, with $x_{0}<\bar{x}<x_{0}+a, y_{0}<\bar{y}<y_{0}+b$, such that $u(\bar{x}, \bar{y})-v(\bar{x}, \bar{y}) \neq 0$.

Consider the (non-empty) set of zeros of the function $u-v$ on the (finite) closed rectangle $x_{0} \leqq x \leqq \bar{x}, y_{0} \leqq y \leqq \bar{y}$. The following subset, eall it $Z$, of the set of all these zeros of the function $u-v$ will be of interest. Let $Z$ be the set of all points $\left(\xi_{0}, \eta_{0}\right)$ of the rectangle $x_{0} \leqq x \leqq \bar{x}, y_{0} \leqq y \leqq \bar{y}$ having the property that the function $u-v$ has the value zero at every point $(x, y)$ such that $x_{0} \leqq x \leqq \xi_{0}, y_{0} \leqq y \leqq \eta_{0}$. The set $Z$ is not empty, since the point $\left(x_{0}, y_{0}\right)$ is itself an element of the set. It is readily seen that the set $Z$ is closed, i. e. that if the sequence of points $\left(x_{1}, y_{1}\right),\left(x_{2}, y_{2}\right),\left(x_{3}, y_{3}\right), \ldots$ of the set $Z$ has the property that $\lim _{n \rightarrow \infty} x_{n}=x^{*}$, and $\lim _{n \rightarrow \infty} y_{n}=y^{*}$, then the limit point $\left(x^{*}, y^{*}\right)$ also belongs to $Z$. (Because, whenever $(x, y)$ satisties the inequalities $x_{0} \leqq x<x^{*}, y_{0} \leqq y<y^{*}$, then there is at least one positive integer $N$, depending on $(x, y)$, such that $x_{0} \leqq x<x_{N}, y_{0} \leqq y<y_{N}$; and hence $u(x, y)-v(x, y)$ must be zero, since $\left(x_{N}, y_{N}\right)$ is a point of $Z$. But then $u(x, y)-v(x, y)$ has the value zero whenever $x_{0} \leqq x<x^{*}, y_{0} \leqq y<y^{*}$; and, by the continuity of $u-v$, the same must be true for $x_{0} \leqq x \leqq x^{*}, y_{0} \leqq y \leqq y^{*}$ ). Therefore, $Z$ is a closed and bounded subset of the finite closed rectangle $x_{0} \leqq x \leqq \bar{x}, y_{0} \leqq y \leqq \bar{y}$. Consequently, the continuous real valued function which has the value $x+y$ for each point $(x, y)$ possesses, when considered only over the set $Z$, a finite maximum on $Z$, which is attained at a point, call it $(\xi, \eta)$. of the set $Z$.

Since the function $u-v$ vanishes at $(\xi, \eta)$, but does not vanish at $(\bar{x}, \bar{y})$, it follows that either $\xi<\bar{x}$ or $\eta<\bar{y}$. For definiteness, suppose first that $\xi<\bar{x}$; the argument will be similar if the other alternative holds. Hence, whenever $\lambda>0$ is sufficiently small, one has that $\xi+\lambda<\bar{x}$. Choose $\lambda>0$ sufficiently small that both $\xi+\lambda<\bar{x}$ and that $\lambda b L<1$, where $L$ is the LIPSCHrTz constant for $f$, and consider the function $u-v$ on the finite closed rectangle consisting of all points $(x, y)$ such that $\xi \leqq x \leqq \xi+\lambda, y_{0} \leqq y \leqq \eta$. The function $u-v$ cannot vanish identically on this rectangle (for if it did then it would vanish identically on the rectangle $x_{0} \leqq x \leqq \xi+\lambda, y_{0} \leqq y \leqq \eta$. and thus the point $(\xi+\lambda, \eta)$ would also belong to $Z$; but $(\xi+\lambda)+\eta>\xi+\eta$, contradicting the choice of $(\xi, \eta)$ as a point of $Z$ for which $x+y$ is a maximum. Therefore, the absolute value function $|u-v|$ has a positive maximum over the rectangle $\xi \leqq x \leqq \xi+\lambda, y_{0} \leqq y \leqq \eta$, at a point $\left(\xi_{m}, \eta_{m}\right)$. (A simply drawn figure will make the following reasoning easier to see). It should be noticed that the function $u-v$ has the value zero at every point $(\xi, y)$ with $y_{0} \leqq y \leqq \eta$; and, of course, at every point $\left(x, y_{0}\right)$ with $\xi \leqq x \leqq \xi+\lambda$. Thus, $\xi<\xi_{m} \leqq \xi+\lambda$ and $y_{0}<\eta_{m} \leqq \eta$.

One has, by the ordinary mean value theorem of the differential calculus, 
applied to the continuous difference function $u\left(x, \eta_{i}\right)-v\left(x, \eta_{m}\right)$ on the $x$ interval $\xi \leqq x \leqq \xi_{m}$ (recall that $u\left(\xi, \eta_{m}\right)-v\left(\xi, \eta_{m}\right)=0$ ), that

$$
u\left(\xi_{m}, \eta_{m}\right)-v\left(\xi_{m}, \eta_{m}\right)=\left(\xi_{m}-\xi\right)\left[u_{\infty}\left(\xi^{*}, \eta_{m}\right)-v\left(\xi^{*}, \eta_{m}\right)\right]
$$

where $\xi<\xi^{*}<\xi_{m}$. Now, applying the mean value theorem of the differential calculus to the continuous difference function $u_{x}\left(\xi^{*}, y\right)-v_{x}\left(\xi^{*}, y\right)$ on the $y$ interval $y_{0} \leqq y \leqq \eta_{m}$; one obtains (recall that $u_{x}\left(\xi^{*}, y_{0}\right)-v_{x}\left(\xi^{*}, y_{0}\right)=0$ ),

$$
u\left(\xi_{m}, \eta_{m}\right)-v\left(\xi_{m}, \eta_{m}\right)=\left(\xi_{m}-\xi\right)\left(\eta_{m}-y_{0}\right)\left[u_{x y}\left(\xi^{*}, \eta^{*}\right)-v\left(\xi^{*}, \gamma_{i}^{*}\right)\right],
$$

where $\xi<\xi^{*}<\xi_{m}$, and $y_{0}<\eta^{*}<\eta_{m}$; and thus

$$
\left|u\left(\xi_{m}, \eta_{m}\right)-v\left(\xi_{m}, \eta_{m}\right)\right| \leqq \lambda b\left|u_{x y}\left(\xi^{*}, \eta^{*}\right)-v_{x y}\left(\xi^{*}, \eta^{*}\right)\right| .
$$

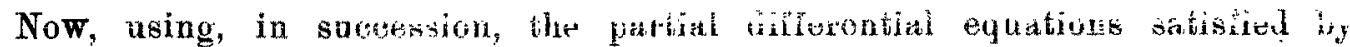
$u(x, y)$ and $v(x, y)$ in the open rutungle $x_{0}<x<x_{0}+a, y_{0}<y<y_{0}+a$;

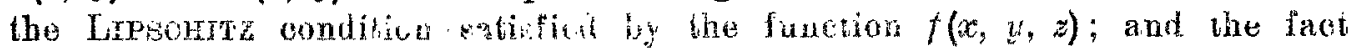

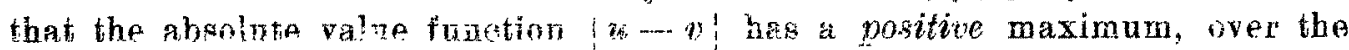
rectangle $\xi \leq x \leq \xi+\lambda, y_{0} \leq y \leq \eta$, at the point $\left(\xi_{m}, \eta_{m}\right)$, one obtains from the very last inequality that

$$
\begin{aligned}
0<\left|u\left(\xi_{m}, \eta_{m}\right)-v\left(\xi_{m}, \eta_{m}\right)\right| & \leq \lambda b\left|f\left(\xi^{*}, \eta^{*}, u\left(\xi^{*}, \eta_{i}^{*}\right)\right)-f\left(\xi^{*}, \eta^{*}, v\left(\xi^{*}, \eta^{*}\right)\right)\right| \\
& \leqq \lambda b L_{1} \mid u\left(\xi^{*}, \eta^{*}, \ldots \nu\left(\xi^{*}, \eta^{*}\right) \mid\right. \\
& \leqq \lambda b L\left|u\left(\xi_{m}, \eta_{m}\right)-v\left(\xi_{m}, \eta_{l m}\right)\right| .
\end{aligned}
$$

But then it follows, upon division by the positive number $\left|u\left(\xi_{m}, \eta_{m}\right)-v\left(\xi_{m}, \eta_{m}\right)\right|$, that $1 \leqq \lambda b L$, contradicting the initial choice of $\lambda$. The supposition that $\xi<\vec{x}$ has thus been shown to lead to a contradiction.

Suppose, next, that $\eta<\bar{y}$. Hence, whenever $\lambda>0$ is sufficiently small, one has that $\eta+\lambda<\bar{y}$. Choose $\lambda>0$ sufficiently small that both $\eta+\lambda<\bar{y}$ and that $\lambda a L<1$, where $L$ is the Lupsemtz ronstant for $t$, and consider the function $u-v$ on the finite closed restangle consisting of all points $(x, y)$ such that $x_{0} \leqq x \leqq \xi, \eta \leqq y \leqq \eta+\lambda$. The function $u-v$ cannot ranish identically on the rectangle $x_{0} \leqq x \leqq \xi, \eta \leqq y \leqq \eta+\lambda$ (for if it did then it would vanish on $x_{n} \leq x \leq \xi_{y} y=\ldots \eta+\lambda$, and thus the point $(\xi, \eta+\lambda)$ would also belong to $Z$; bat $\xi+(\eta+\lambda)>\xi+\eta$. contradicting the choice of $(\xi, \eta)$ as a point for $Z$ for which $x+y$ is a maximum). Therefore, the absolnce value funotion $|w \cdots v|$ has a positive maximum over the rectangle $x_{0} \leqq x \leqq \xi, \eta \leqq y \leqq \eta+\lambda$, $x^{2}$ a point $\left(\xi_{m}, \eta_{m}\right)$. (A simply drawn figure will make the following reasoning easier to see). It should be noticed that the function $u-v$ has the value zero at every point $(x, \eta)$ with $x_{0} \leqq x \leqq \xi ;$ and, of course, at every point $\left(x_{0}, y\right)$ with $\eta \leqq y \leqq \eta+\lambda$. Thus, $x_{0}<\xi_{m} \leqq \xi$ and $\eta<\eta_{m} \leqq \eta+\lambda$. 
One has, by the ordinary mean value theorem of the differential calculus, applied to the continuous difference function $u\left(x, \eta_{m}\right)-v\left(x, \eta_{m}\right)$ on the $x$ interval $x_{0} \leqq x \leqq \xi_{m}$ (recall that $u\left(x_{0}, \eta_{m}\right)-v\left(x_{0}, \eta_{m}\right)=0$ ), that

$$
u\left(\xi_{m}, \eta_{m}\right)-v\left(\xi_{m}, \eta_{m}\right)=\left(\xi_{m}-x_{0}\right)\left[u_{x}\left(\xi^{*}, \eta_{m}\right)-v_{x}\left(\xi^{*}, \eta_{m}\right)\right]
$$

where $x_{0}<\xi^{*}<\xi_{m}$. Now, applying the mean value theorem of the differential calculus to the continuous difference function $u_{x}\left(\xi^{*}, y\right)-v_{x}\left(\xi^{*}, y\right)$ on the $y$ interval $\eta \leqq y \leqq \eta_{m}$, one obtains (recall that $u_{x}\left(\xi^{*}, \eta\right)-v_{x}\left(\xi^{*}, \eta\right)=0$ ),

$$
u\left(\xi_{m}, \eta_{m}\right)-v\left(\xi_{m}, \eta_{m}\right)=\left(\xi_{m}-x_{0}\right)\left(\eta_{m}-\eta\right)\left[u_{x \nu}\left(\xi^{*}, \eta^{*}\right)-v_{x \nu}\left(\xi^{*}, \eta^{*}\right)\right]
$$

where $x_{0}<\xi^{*}<\xi_{m}$, and $\eta<\eta^{*}<\eta_{m}$, and thus

$$
\left|u\left(\xi_{m}, \eta_{m}\right)-v\left(\xi_{m}, \eta_{m}\right)\right| \leqq \lambda a\left|u_{x y}\left(\xi^{*}, \eta^{*}\right)-v_{x \nu}\left(\xi^{*}, \eta^{*}\right)\right|
$$

Now, using, in succession, the partial differential equations satisfied by $u(x, y)$ and $v(x, y)$ in the open rectangle $x_{0}<x<x_{0}+a, y_{0}<y<y_{0}+b$; the LIPschitz condition satisfied by the function $f(x, y, z)$; and the fact that the absolute value function $|u-v|$ has a positive maximum, over the rectangle $x_{0} \leqq x \leqq \xi, \eta \leqq y \leqq \eta+\lambda$, at the point $\left(\xi_{m}, \eta_{m}\right)$, one obtains from the very last inequality that

$$
\begin{aligned}
0<\left|u\left(\xi_{m}, \eta_{m}\right)-v\left(\xi_{m}, \eta_{m}\right)\right| & \leqq \lambda a\left|f\left(\xi^{*}, \eta^{*}, u\left(\xi^{*}, \eta^{*}\right)\right)-f\left(\xi^{*}, \eta^{*}, v\left(\xi^{*}, \eta^{*}\right)\right)\right| \\
& \leqq \lambda a L\left|u\left(\xi^{*}, \eta^{*}\right)-v\left(\xi^{*}, \eta^{*}\right)\right| \\
& \leqq \lambda a L\left|u\left(\xi_{m}, \eta_{m}\right)-v\left(\xi_{m}, \eta_{m}\right)\right| .
\end{aligned}
$$

But the it follows, upon division by the positive number $\left|u\left(\xi_{m}, \eta_{m}\right)-v\left(\xi_{m}, \eta_{m}\right)\right|$, that $1 \lambda a L$, contradicting the initial choice of $\lambda$. The supposition that $\eta<\bar{y}$ has thus also been shown to lead to a contradiction, and the proof is complete.

The theorem just proved is applicable in cases where theorems 4 and 5 of section 3 are not applicable to the differential equation $u_{x \nu}=f(x . y, u)$. Consider the characteristic vatue problem consisting of the partial differential equation $u_{x y}=c(x, y) u$, where $c(x, y)$ is an aróitrary real valued function defined on $x_{0}<x<x_{0}+a, y_{0}<y<y_{0}+b$, which is, however, bounded in absolute value (i. e. there exists a number $L \geqq 0$ such that $|c(x, y)| \leqq L$ for $\left.x_{0}<x<x_{0}+a, y_{0}<y<y_{0}+b\right)$; and the characteristic conditions $u\left(x_{0}, y\right)=0, u\left(x, y_{0}\right)=0$. The function $u(x, y)=0$ is a solution $;$; it is continuous on the partially closed rectangle $x_{0} \leqq x<x_{0}+a, y_{0} \leqq y<y_{0}+b$, has a finite, continuous $x$ partial derivative on $x_{0}<x<x_{0}+a, y_{0} \leqq y<y_{0}+b$, and a finite $x y$ partial derivative on $x_{0}<x<x_{0}+a, y_{0}<y<y_{0}+b$; and satisfies the partial differential equation on the open rectangle $x_{0}<x<x_{0}+a$, 
$y_{0}<y<y_{0}+b$. Here, $f(x, y, z)=c(x, y) z$, but theorems 3 and 4 of section 3 are not applicable since $c(x, y)$ need not be continuous. On the other hand, theorem 6 still guarantees that there is no other solution.

\section{BIBLIOGRAPHY}

[1] PICARD, E., Sur les méthodes d'approximations successives dans la théorie des équations differentielles, (Note I to vol. 4 of G. Darboux, Lecons sur la Thérie Générale des Surfaces, Paris, 1896, pp. 353-367).

[2] Lеwy, H., Uber das Anfangswertproblem einer hyperbolischen nichtlinearen partiellen Differentialgleichung zweiter Ordnung mit zwei unabhängigen Veränderlichen, "Math. Annalen s, 98, 179-190 (1928).

[3] BнскеRт, H., Existenz- und Eindentigkeitsbeveise für das Differenzen-verfahren zur Losung des Anafangsuertproblems, des gemischten Angangs-Randuert- und des charakteristischen Problems einer hyperbolischen Differentialgleichung zueiter Ordnung mit zwei unabhangigen Variablen. "Ber. Verh. Săchs. Akad. Wiss. Leipzig, Math.-naturwiss. ", Kl. 97, H. 4 (1950).

[4] LeEHEx, $\mathcal{P}_{\text {, }}$ On the existence of not necessarily unique solutions of classical hyperbolic boundary value problems for non-linear second order partial differential equations in two independent variables, $\mathrm{Ph}$. D. thesis, Brown University, June. 1950.

[5] Zwirner, G., Sull'approssimazione degli integrali del sistema differenziale $\partial^{2} z / \partial x \partial y=$ $f(x, y, z), z\left(x_{0}, y\right)=\dot{\psi}(y), z\left(x, y_{0}\right)=\varphi(x)$, * Atti dell'Istituto Veneto di Scienze, Lettere ed Arti, Cl. Sci. Fis. Nat. », 109, 219-231 (1959.51).

[6] Hartman, P. and Wintner, A, On hyperbolic partial differential equations, "American Journal of Mathematics », 74, 834.864 (1952).

[7] Conт, R., Sul problema di Darboux per l'equazione $z_{x y}=f\left(x, y, z, z_{x}, z_{y}\right)$, "Annali dell'Universita di Ferrara», Nuova ser., Sez. 7, Sc. Mat. 2, 129.140 (19503).

[8] Moore, R. H., Proof of an existence and uniqueness theorem of Picard for a non-linear hyperbolic partial differential equation, M. A. thesis, University of Maryland, June, 1955.

[9] Azız, A. K., On higher order boundary value problems for hyperbolic partial differential equations in two and three variables, $\mathrm{Ph}$. D. thesis, University of Maryland, June, 1958.

[10] Cont, R. Sull'equazione integrodifferenziale di Darboux-Picard, "Le Matematiche * 13, $30-39$ (195்).

[11] DiAz, J. B., On an analogue of the Euler-Cauchy polygon method for the numerica. solution of $u_{x y}=f\left(x, y, u, u_{x}, u_{y}\right)$, *Archive for Rational Mechanics and Analysis » 1, 357.390 (1908) (also issued as NAVORD Report 4451, U. S. Naval Ordnance Labo, ratory, White Oak, Maryland, January 16, 1957).

[12] Guglieluino, Francesco, Sulla risoluzione del problema di Darboux per l'equazione $s=f(x, y, z)$, Boll. Unione Mat. Italiana *, (3), vol. 13, 308-318 (1958).

[13] Villari, Gaetano, Su un problema al contorno per una classe di sistemi di eguazione alle devivate parziali, "Boll. Unione Mat. Italiana (3), vol. 13, 1-8 (1958).

[14] Concan, James, The Cauchy problem and the mixed boundary value problem for a nonlinear hyperbolic partial differential equation in two independent variables, 3, 355.380 (1959). (Ph. D. thesis, University of Maryland, June, 1958).

[15] Draz, J. B., and WALteR, W. L., On uniqueness theorems for ordinary differential equations and for partial equations of hyperbolic type, Technical Note BN-177, Uni. 
versity of Maryland. July, 1959; "Transactions of the American Mathematical Socie. ty , vol. $96,1960,90.100$.

[16] Moont, R. H., On approximation of the solutions of the Goursat problem for second order quasi-linear equations, Ph. D. thesis, University of Michigan, June 1959 (to appear in the Archive for Rational Mechanics and Analysis).

[17] Santoro, Paolo, Sul problema di Darboux per l'equazione $s=f(x, y, z, p, q)$ e il fenomeno di Peano, *Rendiconti dell'Accademia Nazionale dei XL", Ser. IV, vol. X (82)* 3-17 (1959).

[18] WALTER W, Eindeutigkeitssatze fuv die Differentialgleichung $u_{x y}=f\left(x, y, u, u_{x}, u_{y}\right)$, - Math. Zeit. *, 71, 398-324 (1959) (see also Technical Note BN-159, University of Maryland, 1960).

[19] Binlings, J. H., Extensions of the Laplace method, Ph. D. thesis, University of Mary, land, June 1960 (see also Techical Note BN-209, University of Maryland, 1960).

[20] Sternberg, H. M., The solution of the characteristic and the Cauchy problems for the Bianchi partial differential equation in $n$ independent variables by a generalization of Riemann's method, Ph. D. thesis, University of Maryland, June 1960.

[21] AzIz, A. K., and DiAz, J. B.. On higher order boundary value problems for a linear. hyperbolic partial differential equation in three independent variables (to appear in the Indiana Journal of Mathematics and Mechanics).

[22] Shanahan, John, P., On uniqueness questions for hyperbolic differential equations (to appear in the Pacific Journal of Mathematics). 